\title{
Proyectos de intervención y gestión humana en sitios de práctica organizacional desarrollados en el marco del programa de psicología de la Universidad Piloto de Colombia
}

\section{Human intervention and management projects in organizational practice sites developed within the framework of the psychology program of the Universidad Piloto de Colombia}

Cómo citar este artículo:

González, L. Y., Rueda, L., \& Santana, G. (2017). Proyectos de intervención y gestión humana en sitios de práctica organizacional desarrollados en el marco del programa de psicología de la Universidad Piloto de Colombia. Revista Enfoques, 2(1). http://dx.doi.org/10.24267/23898798.212

Derechos de autor: Licencia Creative Commons AtribuciónNoComercial-SinDerivadas 4.0 internacional y 2.5 Colombia (CC BYNC-ND 2.5 (C)

Recibido: agosto 14 de 2016

Revisado: agosto 23 de 2017

Aceptado:septiembre 05 de 2017

*Psicólogos M.sc., docentes del programa de psicología Universidad Piloto de Colombia. Contacto: yair-gonzalez@ unipiloto.edu.co, liliana-rueda@unipiloto.edu.co,german-santana@unipiloto.edu.co 
Resumen | Las prácticas profesionales de la Universidad Piloto de Colombia se conciben como una estrategia pedagógica que aproxima a los estudiantes de Psicología a escenarios reales de intervención organizacional, articulando aspectos teóricos, metodológicos y conceptuales en el día a día de una empresa o institución. En ese sentido, la intervención organizacional adelantada por los practicantes debe sustentarse en un espacio de aprendizaje denominado Proyecto integrador el cual permite desarrollar competencias en las dimensiones del saber, el ser y el hacer. Este documento presenta un panorama de ejercicios académicos abordados por estudiantes de décimo nivel y que son más usuales en el área organizacional fortaleciendo la línea tradicional de la psicología organizacional. Por ende, este artículo se propone plantear una reflexión al respecto de los roles y las funciones que desempeñan los practicantes del área de Gestión Humana (Programa de Psicología, U.P.C.) dentro de las organizaciones en las cuales adelantan su labor. De una manera descriptiva se identifican las áreas tradicionales de trabajo en las que el estudiante de último semestre se desenvuelve durante su periodo de práctica, no obstante, al mismo tiempo se señalan otras posibilidades de intervención que pueden realizar en las organizaciones.

Palabras clave I Psicología organizacional, práctica profesional, gestión humana, intervención, espacios de aprendizaje.

Abstract | The professional practices of the Universidad Piloto de Colombia are conceived as a pedagogical strategy that brings students of Psychology closer to real scenarios of organizational intervention, articulating theoretical, methodological and conceptual aspects in the daily life of a company or institution. In this sense, the organizational intervention advanced by the practitioners must be based on a learning space called Project Integrator which allows developing competencies in the dimensions of knowledge, being and doing. This paper presents a panorama of academic exercises addressed by tenth-level students which are more usual in the organizational area by strengthening the traditional lines of organizational psychology. Therefore, this article proposes to reflect on the roles and functions of practitioners in the area of Human Management (Psychology Program, U.P.C.) within the organizations in which they advance their work. In a descriptive way, the traditional areas of work are identified in which the student of last semester develops during his period of practice, however, at the same time other possibilities of intervention that can be realized in the organizations are indicated.

Keywords / organizational psychology, professional practice, human management, intervention, learning spaces.

$\boldsymbol{\epsilon} \| 120$ 


\section{Introducción}

En el curso de su práctica profesional, los estudiantes de 10 nivel del programa de Psicología de la Universidad Piloto de Colombia deben formular un documento denominado Proyecto integrador ${ }^{1}$. Este contiene el plan de acción de la intervención adelantada por los practicantes adscritos al área de Gestión humana y plasma, por ende, aquellas acciones y estrategias que el futuro psicólogo plantea, promueve y desarrolla frente a una problemática particular de la organización en donde se encuentra. Asimismo, brinda la posibilidad de poner en práctica lo aprendido durante todo su proceso de formación.

Este Proyecto se lleva a cabo a lo largo de seis meses calendario; tiempo en el que tiene la asesoría de profesores del área organizacional quienes orientan al practicante semana tras semana, contando con una guía de desarrollo denominada Rúbrica de intervención organizacional.

Dicha Rúbrica está compuesta por tres partes: la primera corresponde a una descripción donde se muestran la historia, misión y visión de la organización en donde se encuentra el practicante. Adicionalmente, en esta parte debe diseñarse, junto con el jefe inmediato, un cronograma de acción-intervención que cuenta con un responsable por parte de la organización y que, al mismo tiempo, es acompañado por el supervisor de la práctica-quien es un docente con experiencia en el área aplicada de la Psicología organizacional y es asignado por la Universidad a cada estudiante matriculado-.

Para desarrollar el Proyecto integrador se identifica un fenómeno de estudio en las organizaciones, se traza un objetivo general y unos objetivos específicos que deben ajustarse al tiempo programado en el cronograma establecido. Sin embargo, la mayoría de los proyectos tienen continuidad por cuanto en seis meses calendario no necesariamente se logran consolidar proyectos de mediano o largo plazo. En consecuencia, este puede tener una primera parte elaborada por el practicante en cuestión y una segunda o tercera parte formulada por quien(es) lo suceda(n) o por otro miembro de la organización.

\footnotetext{
${ }^{1}$ Proyecto Integrador. Asignatura perteneciente a décimo nivel de formación y corresponde a la malla curricular vigente en el programa de psicología de la Universidad Piloto de Colombia.
} 
En la segunda parte de la Rúbrica, el psicólogo en formación debe plantear un marco teórico que soporte su actuar y su quehacer dentro de la organización. Este marco teórico debe caracterizarse por su puntualidad y por centrarse en los aspectos clave que sirven como base para estructurar el Proyecto. Es relevante mencionar que tanto el supervisor designado por la organización como el docente encargado por la Universidad, tienen conocimiento del desarrollo y de cada una de las partes de la Rúbrica. En otras palabras, el Proyecto integrador se realiza con el concurso de tres partes fundamentales: la organización en donde se realiza la intervención; la Universidad, quien soporta la metodología y la elaboración conceptual, y los desafíos propios de la realidad en la cual está inmerso el practicante de psicología. Esta perspectiva brinda las coordenadas para el despliegue de las dinámicas concretas de formación en el programa, donde se espera que el profesor se asuma como generador de ambientes para el desarrollo integral -como facilitador de autoaprendizajes y co-constructor del saber científico- que promueva en los estudiantes la tarea de construirse a sí mismos y de apropiarse del conocimiento para su aplicación contextualizada. (PEP, 2010)

En su tercera parte, la Rúbrica consigna los resultados propios de la intervención realizada por el practicante. Tal proceso se adelanta una vez la organización y la Universidad han participado en su elaboración, es decir, cuando cada uno de los supervisores ha adelantado su propio proceso de revisión y aprobación. De acuerdo con lo anterior, el Proyecto integrador da cuenta de la unión de distintos actores que buscan abordar una realidad problemática. Es así como desde la experiencia vivida en varias organizaciones por los practicantes de Psicología de 10 nivel en el área de Gestión humana, se presenta a continuación un panorama de empresas del sector servicios en donde se efectuó la práctica organizacional durante el primer y segundo periodo de 2015.

\section{Competitividad y competencias}

La gráfica 1 presenta comparativamente la necesidad sentida por las organizaciones de investigar e intervenir en sus diferentes realidades desde la óptica de las competencias, siendo este tema el de mayor frecuencia en los trabajos efectuados por los practicantes (48\%). Como lo manifiesta Peiró (1999), el incremento en la competitividad hace que las organizaciones respondan con una estructura cada vez menos burocrática y más flexible, lo que impone al 
trabajador nuevos retos en términos de autonomía y versatilidad frente a su conocimiento y sus habilidades para encarar nuevas demandas de servicio.

Gráfica 1. | Porcentaje de proyectos adelantados en 2015 por practicantes de Psicología, U.P.C.

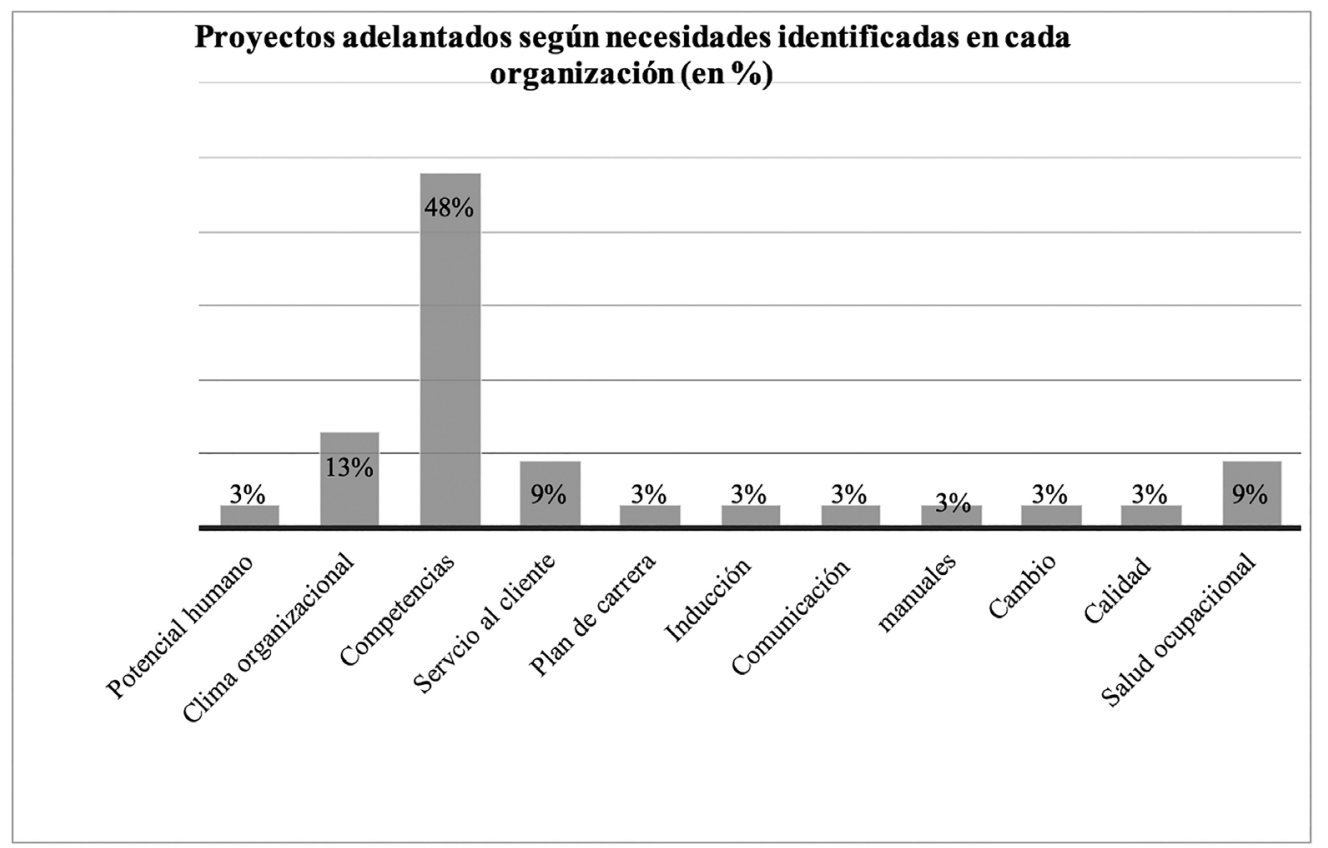

Fuente: Datos suministrados por practicantes en 2015-I y 2015-II. Facultad de Ciencias Humanas Programa de Psicología, U.P.C.

Frente a este primer elemento señalado por Peiró -<el conocimiento >-, el psicólogo(a) pasó de tener nociones o conceptos de orden general, con una serie de teorías universales, a un conocimiento cada vez más interdisciplinar y abierto con una continua reestructuración. Es decir, las organizaciones necesitan cada vez menos de elementos teórico-disciplinares aislados y más de conocimientos complementarios que cambien y se adapten en escalas de tiempo menores. 
Al respecto del segundo elemento mencionado -<habilidad $>-$, esta ya no se limita solamente a una técnica aprendida por repetición en la que por ejemplo se hacen entrevistas de selección con un orden particular; sino corresponde a la habilidad de leer el entorno organizacional en un sentido complejo. Al mismo tiempo, la respuesta a ese entorno debe darse de manera sencilla y práctica, lo cual supone un importante reto. Todo esto, debido a que día a día la competitividad se hace más fuerte y las organizaciones se encuentran cada vez más preparadas para asumir el reto de una economía globalizada.

Aunado a lo anterior, y como lo plantean Peiró, Prieto y Roe (1996), citados por Peiró (1999, p. 169):

[...] las organizaciones tienden a ser cada vez de menor tamaño; los enfoques de gestión humana estarán orientados hacia la racionalización, el adelgazamiento de las organizaciones, trabajo en grupo, organización en grupos semiautónomos o autoregulados, de forma que dicha regulación sea manejada por el mismo grupo en busca de mayor autoeficacia.

Dichas características forman parte del proceso de cambio que han tenido las organizaciones durante los últimos años. Si bien con las anteriores transformaciones se puede aumentar la eficacia, es sobre el trabajador en quien recae el mayor peso y la responsabilidad en su labor, pues le demandan un mayor potencial de innovación, más y mejor capacitación profesional y la capacidad de poder ejercer varios cargos o roles al mismo tiempo, lo cual sugiere que los límites de los puestos de trabajo se han venido disolviendo. Pero de la velocidad de ajuste tanto del trabajador como de la organización a estos cambios, tenderán a multiplicarse sus esfuerzos. Este quizá es uno de los temas de los que se ocupa con más interés la Psicología organizacional: <cómo responder a dichas demandas del medio, sin que sea el trabajador quien termine afectándose con una sobrecarga de trabajo>, pero no resulta fácil. Tal como se expone en este artículo, son algunas organizaciones las que piden al área de Gestión humana del programa de Psicología (U.P.C.) revisar particularmente dicha situación, aún cuando son actores involucrados directamente con dicha problemática. 
Cabe señalar entonces que en Colombia existe desde el año 2003 una reglamentación para que las:

[...] empresas, organizaciones, instituciones educativas o de salud que tengan un número de trabajadores igual o superior a 30, contraten el servicio de psicólogos profesionales, con el objeto de propiciar e implementar políticas de desarrollo humano, bienestar integral y calidad de vida de los mismos trabajadores. (Ley 064 de 2003) ${ }^{2}$

Pero como lo afirman Enciso y Toro (2015, p9.):

En algunos casos, el psicólogo empleado termina preocupándose primordialmente por la conservación de su trabajo, cumpliendo las expectativas de la empresa, supeditando su profesionalismo a las exigencias de rendimiento del factor humano, al precio que sea y atentando en muchas ocasiones contra la responsabilidad social, parte fundamental en la ética del psicólogo. Además, el psicólogo como persona resulta también gravemente vulnerado en el cumplimiento de su proyecto existencial.

Por ende, es clave decir que cuando se habla de las empresas en donde los estudiantes de Psicología realizan sus prácticas, a la vez se habla de ellos -los practicantes- como sujetos que afrontan de una u otra manera una situación similar a la del resto de trabajadores. Además, tienen un doble desafío, pues aparte de cumplir funciones administrativas deben liderar con el área que gestiona lo humano, componente esencial y quizás el más complejo de las organizaciones.

Tradicionalmente los departamentos de gestión humana reciben denominaciones como: $R e$ cursos humanos, Gestión humana o Talento humano, entre otros, sin embargo, las diferencias sustanciales entre una u otra denominación son las funciones propias entre administración y gestión organizacional. En la actualidad ya no es tan rígido el funcionamiento en el seno de las organizaciones como lo era en décadas anteriores; es así como los profesionales en Psicología

\footnotetext{
${ }^{2}$ Por medio de esta Ley "se modifica la reglamentación del ejercicio de la profesión de psicólogo y se dictan otras disposiciones".
} 
han pasado de tener tareas regularmente administrativas, a ser consultores internos de las propias organizaciones, promotores de cambio en cultura organizacional, directores o líderes de nuevas áreas o departamentos; algunos de ellos como el de responsabilidad social empresarial. Pero al mismo tiempo, auditores de calidad, líderes en resolución de conflictos o intermediarios entre Estado, empresa y ciudadanía, por ejemplo. Esto hace que la competitividad esté presente en otras áreas y niveles de la organización.

En este sentido, la formación a nivel de pregrado y posgrado de los psicólogos ha venido variando. Quienes optan por el ámbito de lo organizacional deben tener claro que al trabajar cada vez más en equipos interdisciplinarios y en áreas de la empresa distintas a la de gestión humana, temas como finanzas, mercadeo, administración de negocios, derecho internacional, derecho tributario, política pública, etc., están a la orden del día.

Adicionalmente, en un contexto cada vez más interconectado y atravesado por lo que Castells (1998) denominó la sociedad red, tener conocimientos en dichas áreas se hace imprescindible. Por otra parte, uno de los nodos más importantes de la sociedad actual es la organización o "espacio de trabajo", pues es allí donde suceden y emergen relaciones de orden laboral, social y emocional.

Este "nodo" -organización- es un lugar donde se está la mayor parte de la vida. Por tanto, son las organizaciones y la competitividad el rasgo distintivo de la sociedad capitalista actual. En consecuencia, para abordar este fenómeno la Psicología organizacional puede hacerlo desde diferentes procesos o lógicas, sin embargo, la más fuerte es la productividad empresarial, la cual está ligada a métodos que aseguran mayor rentabilidad. Pero también puede abordarse desde perspectivas críticas que tienen en cuenta otros aspectos diferentes a la racionalidad, como la solidaridad, en donde el núcleo productivo cambia la competitividad por el trabajo mancomunado y cada vez más social. Justamente, el papel que juega tanto la disciplina psicológica o el profesional mismo en una organización, se suscita en realidades complejas que requieren miradas alternas a las tradicionales. Esto significa que, en algunas situaciones, y por cumplir estándares de productividad, un trabajador de la organización y su dimensión humana se pueden ver afectados por cargas excesivas de tareas, maltrato laboral, lógicas de precarización, etc., que en alguna medida se relacionan con medidas o procesos legitimados o avalados por áreas de "gestión humana".

E $\| 126$ 
Dependerá entonces del enfoque que tengan tanto la organización como los profesionales de Psicología que trabajen allí optar por la "competitividad o la solidaridad". En la mayoría de los proyectos que abordaron los estudiantes de la práctica organizacional, la postura adoptada para su intervención se corresponde con una mirada tradicional, es decir, desde la competencia como un rasgo que subyace al individuo y que está relacionado con un estándar de efectividad (Alles, 2002)

\section{Clima organizacional}

En segundo lugar, de la gráfica N.1, se encuentra clima organizacional con un 13\%. Tal como lo señala Pérez de Maldonado, Maldonado y Bustamante (2006) ... el proceso que se lleva a cabo responde a una metodología sistemática y rigurosa, descrito en los siguientes pasos: contextualización, evaluación, diagnóstico e intervención, y los resultados presentan básicamente dos situaciones cuando se aborda el tema de clima organizacional.

La primera en la que los métodos o formas de recolectar la información es lo más importante proviene mayoritariamente de instrumentos cuantitativos, éstos son variados y son paquetes que las empresas o compañías compran en el mercado, en donde se aplica un instrumento con una muestra representativa de los miembros de la organización, que por lo regular es del 40 al 60\%. Pero no tiene la posibilidad de revisar de manera detallada respuestas, actitudes, comportamientos, propuestas de mejora, conversaciones formales o informales y demás información que se puede recolectar y analizar de manera cualitativa; en donde por medio de observaciones participantes, entrevistas, grupos focales, se puede no solo identificar aspectos que intervienen en el clima organizacional, sino alternativas para abordar la problemática de manera participativa.

Con base en lo anterior existe una lógica que subyace a esta dinámica de medición de clima organizacional, y es el cambio. Las organizaciones al no ser estáticas dan cuenta que se relacionan con un mundo que por lógica no lo es. Pero aceptar dicho cambio e incorporarlo dentro de las políticas de una organización y actuar con base en él, es algo que no siempre se hace de manera rápida y oportuna. De esta forma, si se está preparado para aceptar el cambio dentro y fuera de una organización, las mediciones de clima, serán mucho más provecho- 
sas, pero ciertas lógicas dominantes en las empresas u organizaciones son resistentes a que el cambio suceda pues resultan beneficiadas de mantener el control de procesos o de recursos, Zimmermann (2000)

Precisamente son más que acciones de los trabajadores frente a las condiciones de trabajo; constituyen las percepciones, imaginarios y respuestas de los trabajadores ante una situación real, unas condiciones particulares, unas problemáticas específicas, que son vividas día a día por los mismos miembros de una organización o empresa. Pero al involucrar al empleado o miembro de dicha organización no solo como objeto de medición sino como agente activo de cambio, se consigue mayor validez y legitimidad en las gestiones para abordar las distintas problemáticas que hacen parte inherente a toda organización humana, Chiavenato (2009) Es al mismo tiempo una oportunidad para dignificar al trabajador, que como tal merece ser tratado de una manera más cálida que simplemente el receptor de un mail, o de una citación para responder a un cuestionario en donde es solo un número en la estadística de medición de clima organizacional.

Para finalizar este apartado, tal como lo presenta Pérez de Maldonado, Maldonado y Bustamante (2006) un buen clima organizacional, no es producto de la casualidad, es el resultado de una gestión bien concebida y dirigida y, por tanto, cualquier presencia o ausencia de elementos negativos, es consecuencia directa de lo que haga o deje de hacer la gerencia en todos los niveles.

\section{Bienestar y Salud ocupacional}

Por medio de la Ley 100 de 1993 y del Decreto Ley 1295 de 1994 se creó el Sistema General de Riesgos Profesionales; por medio del cual se estableció un modelo de aseguramiento privado de los riesgos ocupacionales y cuyo principal objetivo fue la creación y promoción de una cultura de prevención en accidentes de trabajo y enfermedades profesionales. Lizarazo, Fajardo, Berrio y Quintana (2015) argumentan que al entrar en vigencia dicha ley, se disponía de un sistema enfocado hacia la reparación de daños más que en la prevención, de forma que se hacía énfasis en los modelos de atención médica (es decir, diagnóstico y tratamiento), pensiones por incapacidad, e indemnizaciones, entre otras. Estas funciones eran prestadas 
por el Instituto Seguro Social (ISS), una entidad estatal, único organismo autorizado para estos servicios".

Bajo esta ley, nacen las Administradoras de Riesgos Profesionales (ARP), las cuales se encargan de realizar actividades de prevención, asesoría y evaluación de riegos profesionales, al igual que la prestación de servicios de salud y pago de prestaciones económicas a sus afiliados. La atención médica es usualmente subcontratada a instituciones prestadoras de servicios de salud (IPS) que, junto con las entidades promotoras de salud (EPS) son, en algunos casos, unidades de negocio de una misma entidad. Pero la perspectiva de la realidad contextual que presentan los anteriores autores no es la mejor, pues se necesita reforzar la exigencia en el cumplimiento de las normas, empezando por la afiliación al sistema, la cual no alcanza el 30\% de la cobertura de la población económicamente activa. Según estos autores en Colombia la tasa de desempleo se ha mantenido en los últimos años alrededor del $12 \%$. Por tal motivo se da prioridad a la creación y sostenibilidad del empleo, que a mejorar la calidad de vida laboral a través de mejores sistemas de control y protección de los riesgos ocupacionales.

Teniendo en cuenta el contexto de la economía colombiana, ésta se orienta en mayor medida al aprovechamiento de recursos naturales, así como del sector minero. El sector manufacturero en crecimiento y el uso de mano de obra extensivo, debido a su relativo bajo costo hacen de la empleabilidad en pequeña y mediana industria un sector menos favorecido. Se suma a lo anterior, el hecho de que cerca del $99 \%$ de los establecimientos industriales son clasificados como microempresas (menos de 10 trabajadores), pequeñas (11-50 trabajadores) y medianas (51-200 trabajadores), las cuales generan el $63 \%$ del empleo y el $53 \%$ de la producción bruta del país, lo cual deja un porcentaje de más del $53 \%$ de la economía en manos de la informalidad.

Lo anterior, supone revisar prácticas o procedimientos que llegan a ser casi cotidianos o normales, y que se asocian con "bienestar" como celebración de cumpleaños, viajes regionales, bonos de regalo y planes de incentivo por meta cumplida. Precisamente la referencia de los practicantes de psicología que adelantaron su proyecto integrador en el primer y segundo semestre de 2015 da cuenta que en las organizaciones en donde se llevó a cabo una intervención en temas de salud ocupacional, se supeditaba a este tipo de acciones en donde el "bien- 
estar" es comprendido solo desde la mirada de la recompensa o de acciones recreativas; pero no desde la de los significados frente a la labor que desempeña el trabajador o construcciones sociales de los trabajadores respecto a su actividad diaria. Tal como lo propone en la actualidad la Psicología Positiva cuyo objetivo es justamente catalizar este cambio de la psicología, hacia el desarrollo de las fortalezas de las personas. (Seligman \& Christopher, 2000). Citado por Contreras, (2006).

Este tipo de relación de un miembro de la organización con su entorno laboral, "cómo se siente" qué tan cercana y propia percibe y reconoce a la organización, qué entiende por su labor, son aspectos que no son tenidos en cuenta en la mayoría de las organizaciones. Lo anterior puede deberse a una normalización de procedimientos administrativos que buscan aumentar la productividad por medio de las recompensas sacrificando el bienestar, lo que puede estar relacionado directamente con una cultura organizacional que prioriza la eficiencia a costa de lo humano, pero es muy difícil encontrar las dos dimensiones trabajando conjuntamente. Calderón, Murillo y Torres (2003)

En relación con salud ocupacional, lo referido en los proyectos de práctica, hacen alusión a procedimientos regidos por lógicas de las ARP en las que "pausas activas" chequeos y controles médicos, son hechos más como un aspecto de control de variables médicas que por una idea de salud mental o psicológica. Es decir que la premisa que parece ser la más recurrente en las organizaciones frente a la salud, es la ausencia de enfermedad incapacitante. Pero se olvida lo particular de cada trabajador o miembro de la empresa, asociación o compañía, y son aquellos aspectos de su vida en pareja, de su vida en familia, de su vida social, lo que puede hacer que tenga repercusiones en su salud o en la salud de su círculo cercano.

En resumen, la tradición de la psicología organizacional ha sido separar por esferas lo netamente laboral de la vida diaria, como si separar lo emocional, lo privado y lo familiar fuese un asunto de decisión personal. De ahí la idea de transformar las dinámicas internas y externas de las empresas, las instituciones y las organizaciones a través de propuestas innovadoras desde lo académico modificando los ambientes laborales con el fin de propiciar ambientes laborales que promuevan la calidad de vida y el bienestar psicológico de las personas y sus familias, estableciendo metas realistas de cambio y transformación 
La estrategia didáctica Proyectos Integrados busca transformar la mirada tradicional de bienestar, y resalta que subyace a la misma actividad y labor ejercida. Por tanto, si no se dimensionan estos aspectos, la psicología organizacional desconocería un factor elemental para el ser humano y es que el bienestar está compuesto también por factores subjetivos.

\section{Servicio al cliente}

Así como lo propone Aguilar y Vargas (2010), los trabajadores tienen como contenidos del trabajo los relacionados con ser productores, procesadores, distribuidores y operadores de la información. Es por tanto la información un aspecto decisivo a la hora de medir servicio al cliente, ya que por ejemplo el poder comercializar o promocionar una compañía no solo depende de la publicidad hecha sobre un producto o servicio que se preste en una organización, sino que tanto es difundido como un buen producto por parte de quienes lo usaron o lo adquirieron y cómo esos mismos usuarios y compradores, son quienes pueden potencializar de manera autónoma mayor difusión y contenido.

Cómo se aborda una medición o investigación en servicio al cliente, es el factor fundamental para que las organizaciones consoliden estrategias para fortalecer ésta dimensión. Revisando los proyectos adelantados por los estudiantes de último nivel que realizaron la práctica organizacional, se puede visualizar que no se presenta una metodología clara para abordar servicio al cliente, pues en algunos casos, se realiza por medio del análisis de protocolos de servicio, los cuales se orientan a una serie de reglas de cómo tratar al otro bien sea cliente interno o externo. Otras organizaciones optan por capacitar a sus empleados en cómo solucionar algunas quejas frecuentes en sus compañías y otros optan por buscar soportes tecnológicos que les permitan recepcionar quejas del servicio y entregar en menos tiempo algún tipo de solución frente a las mismas.

Significa que la mayoría de las organizaciones, tratan de incorporar modelos o capacitaciones universales en donde los trabajadores terminan memorizando o acoplando formas de relacionarse y de tratar al otro. Pero siguen teniendo dificultades que la organización no logra reconocer, las cuales entorpecen o afectan el trato y el servicio mismo. Un ejemplo de ello, lo constituye empleados de una organización que deben atender al público constantemente, por 
ende, deben ser distintas de las jornadas de trabajo en tiempo dedicado a la tarea, de los que no tienen contacto con clientes

\section{Conclusiones}

En síntesis, los cuatro temas de mayor investigación e intervención realizados en diferentes organizaciones por los practicantes de 10 nivel adscritos al área de Gestión humana del programa de Psicología de la U.P.C. durante el año 2015, fueron en orden porcentual: Competitividad (48\%), seguida por Clima organizacional (13\%), Salud y Bienestar ocupacional (9\%) y Servicio al cliente (9\%).

Lo anterior permite reconocer que en las compañías en donde se realizó la práctica organizacional, existe una mayor demanda de investigación en temas que tienen que ver con formación y evaluación por competencias. Esto puede explicarse por un contexto de mercado que exige cada vez más que las organizaciones lleven a cabo procesos de reestructuración en aras de ser más eficientes para atender el volumen de demanda y la calidad de productos y servicios ofertados y con ello ser competitivas en el sector en el que se encuentren. Pero indistinto de la razón, la diferencia en el peso porcentual entre competitividad y clima organizacional que es el segundo tema de mayor investigación e intervención, hace notar que sigue siendo recurrente en las organizaciones hablar y gestionar con base en competencias, así como desarrollar estrategias para competir con otras organizaciones del sector.

Al mismo tiempo, la competitividad, característica de una economía de mercado neoliberal, imprime dentro de los nuevos perfiles de trabajadores una carga adicional y una exigencia más fuerte como "trabajador del siglo XXI" (discurso ampliamente referido dentro del contexto organizacional, debido al tipo de competencias que refieren las organizaciones en el perfil del trabajador) en dónde se pierden los límites de los roles en las organizaciones, aumenta la autonomía y responsabilidad de los grupos de trabajo, y los conocimientos que se exigen para desempeñar una función, son cada vez más de orden interdisciplinar. Más orientados no hacia referentes conceptuales sino metodologías y abordajes estratégicos que disminuyan los recursos invertidos, aumenten la productividad y necesiten menos tiempo. Lo anterior si bien produce mayor efectividad, puede ocasionar afectación a la calidad de vida del trabajador, su 
salud física y psicológica, lo cual está estrechamente relacionado con el segundo porcentaje en investigación e intervención, que es clima organizacional el cual fue de $13 \%$ en las prácticas de los estudiantes durante el año 2015.

Precisamente se logra identificar, que se ha venido dando un giro hacia metodologías de investigación e intervención más de orden cualitativo, las cuales permiten reconocer o identificar problemáticas en donde los métodos tradicionales como encuestas y aplicación de instrumentos estandarizados han hecho crisis. Esta ventaja de lo cualitativo, permite reconocer que en lo organizacional se está dando una tendencia por abordajes que privilegian la interacción humana, profesional-entorno, profesional-profesional, colaborador-colaborador y no tanto la lógica: trabajador-instrumento, profesional-instrumento.

De ésta manera se vuelve más participativa y proactiva la labor no solo del psicólogo (a) de gestión humana, sino que el colaborador de la organización es incluido, dentro del proceso de diagnóstico de clima de una manera integral; no solo para evaluar sino para tener en cuenta sus recomendaciones, sus propuestas de cambio y su experiencia en el cambio. En resumen, los miembros de la organización no son un objeto de medición sino agentes de cambio y transformación activa.

Esta nueva lógica organizacional hace que el nuevo trabajador de gestión humana, vaya más allá de encuestas y aplicaciones de instrumentos a un mayor nivel de acercamiento e involucramiento, el cual es percibido por el miembro de la organización con quien interactúa como positivo con una ventaja adicional y es la posibilidad de retroalimentar la labor realizada. Esto supone un cambio en el rol del psicólogo (a) pues ya no es un simple instrumentalista, sino un profesional que, bajo un criterio, y una forma de adentrarse en el fenómeno permite analizar con más profundidad la realidad organizacional en la que se encuentra.

En lo referente a salud ocupacional, tercer tema más investigado y abordado por los practicantes de psicología, permite desde una perspectiva crítica, ir más allá de la visión tradicional de prevención de riesgo psicosocial o campañas de promoción y prevención de la salud, y avanzar hacia una comprensión de bienestar y salud en el trabajo como una elaboración subjetiva, construida por los miembros de la organización y no solo como una estandarización de procesos guiados por una ARL (Aseguradora de Riesgos Laborales). 
Para finalizar, el abordaje del <servicio al cliente> en las organizaciones plantea un reto particular debido a que los miembros de una organización al no sentirse satisfechos, cómodos o en unas condiciones de bienestar acordes a la labor que desempeñan, hacen que servicio al cliente se convierta en una condición que deben cumplir, en lugar de una actitud y un proceso de atención y de respuesta equitativo, cordial y que busque la equidad en el trato al otro. Por tanto, los esfuerzos que adelantan las organizaciones en capacitaciones, llamados de atención o implementación de políticas de mejora en servicio al cliente, no son suficientes y necesitan del concurso de actores del departamento de gestión humana que propendan por la mejora sistemática de calidad de vida laboral para que servicio al cliente sea un resultado y no una causa desde la gestión que se realice en las organizaciones.

En conclusión Proyectos integrados como ha sido concebido es un espacio académico que integra la disciplina y las empresas, dando sentido y forma al Proyecto Educativo del Programa de Psicología de la Universidad Piloto de Colombia, el cual plantea que la construcción de conocimiento supone hablar de un proceso articulado al contexto histórico y cultural en el que se desarrolla, y de una función social que determina una concepción de la formación profesional centrada en competencias, fruto de un proceso sistemático de sensibilización a las condiciones de la disciplina y del ejercicio profesional (PEP, 2010).

\section{Referencias}

Aguilar, J. y Vargas, J. (2010) Servicio al cliente. Network de Psicología Organizacional. México: Asociación Oaxaqueña de Psicología A.C.

Alles, M. (2002). Gestión por competencias: El diccionario.Editorial Granica. Buenos Aires Argentina.

Calderón, G., Murillo, S., y Torres, K. (2003). Cultura Organizacional y Bienestar laboral. Cuadernos de Administración, vol. 16, núm. 25, enero-junio, 2003, pp. 109-137 Pontificia Universidad Javeriana Bogóta, Colombia

Castells, M. (1998). La Era de la Información: Economía, Sociedad y Cultura. Alianza Editorial 
Contreras, F., \& Esguerra, G, (2006). Psicología positiva: una nueva perspectiva en psicología. Diversitas: Perspectivas en Psicología, vol. 2, núm. 2, julio-diciembre, 2006, pp. 311-319. Recuperado de http://www. redalyc.org/pdf/679/67920210.pdf

Chiavenato I. (2009). Gestión del Talento Humano. Tercera Edición, Mc Graw Hill. México, D.F.

Diener, E. (1984). Subjective well-being. Psychological Bulletin, 95(3), 542-575.

Enciso Forero, E., \& Perilla Toro, L. (2015). Visión Retrospectiva, Actual y Prospectiva De La Psicología Organizacional. Acta Colombiana De Psicología, O(11), 5-22. Recuperado de:http://editorial.ucatolica.edu.co/ ojsucatolica/revistas_ucatolica/index.php/acta-colombiana-psicologia/article/view/476/473

Lizarazo, C., Fajardo, J., Berrio, S. Y Quintana L. Breve historia de la Salud Ocupacional en Colombia. Departamento de Ingeniería Industrial. Pontificia Universidad Javeriana. Bogotá, Colombia. Recuperado de: http:// www.oiss.org/estrategia/IMG/pdf/Breve_historia_sobre_la_salud_ocupacional_en_Colombia1.pdf. Consultado 2015-03-10

Peiró. J. (1999). Psicología de las organizaciones: Cambios recientes, retos y perspectivas. Revista de Psicología General y Aplicada. Pág 169-176.UIPOT. Unidad de Investigación de Psicología de las Organizaciones y del Trabajo. Área de Psicología Social. Universitat de València.

Pérez de Maldonado, I., Maldonado, M. y Bustamante S. (2006). Clima organizacional y gerencia: inductores del cambio organizacional. Investigación y Postgrado v.21 n.2 Caracas oct.

Proyecto Educativo del Programa. (2010). Programa de Psicología. Universidad Piloto de Colombia.

Zimmermann, A. (2000). Gestión del cambio organizacional. Caminos y herramientas. Ediciones Abya-Yala. QuitoEcuador

\section{Normas, leyes y decretos}

Colombia, (2003). Proyecto De Ley 064 De 2003 Cámara. por la cual se modifica la reglamentación del ejercicio de la profesión de psicólogo y se dictan otras disposiciones. Disponible en http://legislacion.vlex.com.co/ vid/proyecto-ley-ca-mara-451267242?_ga=1.238192768.1223795034.1457915940. Consultado 